\title{
Disability and the COVID-19 Pandemic
}

\author{
Using Twitter to Understand Accessibility during Rapid Societal Transition
}

\author{
Cole Gleason * \\ HCI Institute, Carnegie Mellon \\ University, Pittsburgh, PA, USA \\ cgleason@cs.cmu.edu \\ Jason $\mathrm{Wu}$ \\ HCI Institute, Carnegie Mellon \\ University, Pittsburgh, PA, USA \\ jasonwu2@cs.cmu.edu \\ Jeffrey P. Bigham \\ HCI Institute, Carnegie Mellon \\ University, Pittsburgh, PA, USA \\ jbigham@cs.cmu.edu
}

\author{
Stephanie Valencia* \\ HCI Institute, Carnegie Mellon \\ University, Pittsburgh, PA, USA \\ svalenci@andrew.cmu.edu \\ Anhong Guo \\ HCI Institute, Carnegie Mellon \\ University, Pittsburgh, PA, USA \\ anhongg@cs.cmu.edu \\ Cynthia L. Bennett* \\ HCI Institute, Carnegie Mellon \\ University, Pittsburgh, PA, USA \\ cbennet2@andrew.cmu.edu
}

\author{
Lynn Kirabo \\ HCI Institute, Carnegie Mellon \\ University, Pittsburgh, PA, USA \\ lkirabo@andrew.cmu.edu \\ Elizabeth J. Carter \\ Robotics Institute, Carnegie Mellon \\ University, Pittsburgh, PA, USA \\ lizcarter@cmu.edu \\ Amy Pavel* \\ HCI Institute, Carnegie Mellon \\ University, Pittsburgh, PA, USA \\ apavel@cs.cmu.edu
}

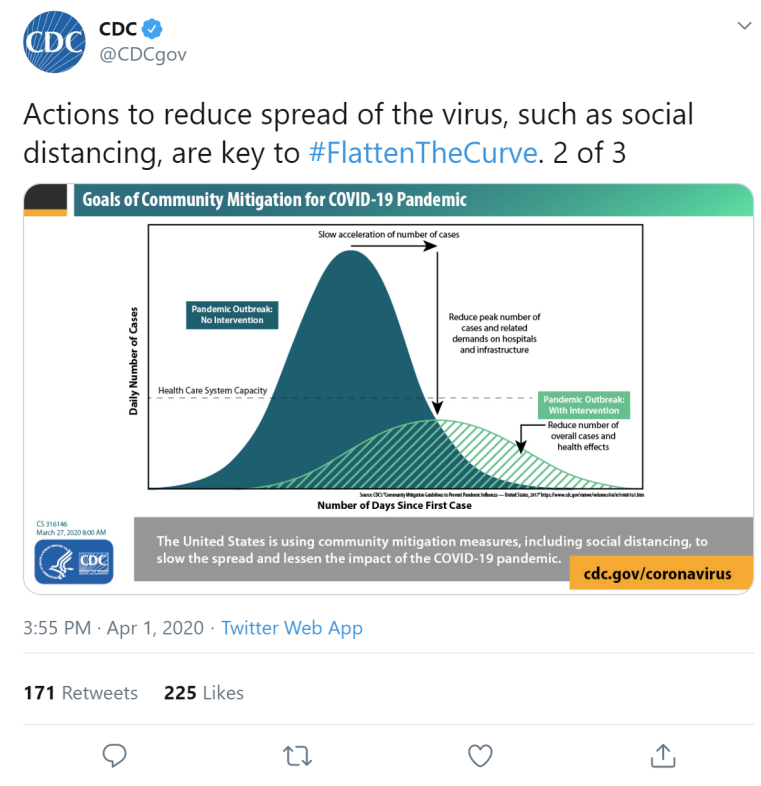

Figure 1: This inaccessible tweet posted by the U.S. Centers for Disease Control contains the common public health infographic about "flattening the curve." These images were posted without alternative text, leaving them incomprehensible to screen reader users.

"Cole Gleason and Stephanie Valencia contributed equally to this work. Cynthia L. Bennett and Amy Pavel contributed equally to this work.

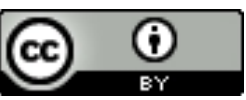

This work is licensed under a Creative Commons Attribution International 4.0 License. ASSETS '20, October 26-28, 2020, Virtual Event, Greece

(C) 2020 Copyright held by the owner/author(s).

ACM ISBN 978-1-4503-7103-2/20/10

https://doi.org/10.1145/3373625.3417023

\section{ABSTRACT}

The COVID-19 pandemic has forced institutions to rapidly alter their behavior, which typically has disproportionate negative effects on people with disabilities as accessibility is overlooked. To investigate these issues, we analyzed Twitter data to examine accessibility problems surfaced by the crisis. We identified three key domains at the intersection of accessibility and technology: (i) the allocation of product delivery services, (ii) the transition to remote education, and (iii) the dissemination of public health information. We found that essential retailers expanded their high-risk customer 
shopping hours and pick-up and delivery services, but individuals with disabilities still lacked necessary access to goods and services. Long-experienced access barriers to online education were exacerbated by the abrupt transition of in-person to remote instruction. Finally, public health messaging has been inconsistent and inaccessible, which is unacceptable during a rapidly-evolving crisis. We argue that organizations should create flexible, accessible technology and policies in calm times to be adaptable in times of crisis to serve individuals with diverse needs.

\section{CCS CONCEPTS}

- Human-centered computing $\rightarrow$ Accessibility; • Social and professional topics $\rightarrow$ User characteristics; People with disabilities.

\section{KEYWORDS}

Accessibility, Disability, Twitter, Social Media, COVID-19, Emergency, Delivery Services, Online Education, Public Health

\section{ACM Reference Format:}

Cole Gleason, Stephanie Valencia*, Lynn Kirabo, Jason Wu, Anhong Guo, Elizabeth J. Carter, Jeffrey P. Bigham, Cynthia L. Bennett*, and Amy Pavel* . 2020. Disability and the COVID-19 Pandemic: Using Twitter to Understand Accessibility during Rapid Societal Transition. In The 22nd International ACM SIGACCESS Conference on Computers and Accessibility (ASSETS '20), October 26-28, 2020, Virtual Event, Greece. ACM, New York, NY, USA, 14 pages. https://doi.org/10.1145/3373625.3417023

\section{INTRODUCTION}

As the onset of the COVID-19 pandemic motivated public health statements, widespread stay-at-home and shelter-in-place orders, and a broad transition from in-person to online interactions, dramatic transitions occurred that affected people's lives. Individuals, organizations, businesses, and governments have been forced into new and unknown circumstances and have had to adapt rapidly. Throughout any rapid change, people with disabilities (PWD) feel a disproportionate impact of new policies and behaviors, sometimes as a result of unintended consequences [81]. PWD are also uniquely poised to understand and critique these new policies and ways of life because of their extensive lived experiences negotiating health precarity and roles as longtime advocates of some tools now widely used to properly social distance.

In this research, we characterize the impact of these effects across multiple domains using information from social media posts, organization websites, and news outlets. As lockdowns and other stay-at-home orders proliferated in late March and early April of 2020, we did preliminary reading on how PWD and high-risk individuals were affected. Using posts from Twitter, we identified major topics of discussion and focused on three key domains where technology and disability intersect in this crisis: product delivery, remote education, and public health information dissemination. We obtained a broad set of perspectives about the impact of recent changes by conducting domain-specific Twitter searches and analyses. We added additional context to these perspectives through follow-up analyses of specific sectors, including the prioritization policies of grocery stores, the accessibility of major online education platforms, and the presence of alternative text on public health department tweets.

While services like online ordering and grocery delivery existed before the COVID-19 outbreak, the use of these services spiked dramatically as social distancing policies proliferated [11]. Product delivery and online ordering have been a boon and a pain point for PWD for decades [12, 28, 49, 64]. However, flooding of these services when the wider population needs or wants them (due to convenience, business closures, etc.) has resulted in reports of unavailability and/or delays for all users (e.g., $[3,67]$ ). For PWD or other at-risk individuals who may not have other options, this adds a level of inconvenience or even danger to previously quotidian experiences like grocery shopping. Our analysis of tweets related to this domain revealed criticism of access to groceries, both inperson at stores and through online ordering. We corroborated these accounts by examining the policies of the largest grocery store chains in the US and UK, finding that only two offered any prioritization of delivery slots for high-risk individuals. Even still, these policies struggled to target the appropriate users, as it can be difficult to determine who is in most need of a limited service. Social distancing regulations have highlighted the importance of delivery services for everyone and often expanded their availability; however, inconsistent attention to the needs of PWD led to insufficient support.

Online education has shifted from being one potential choice to being the only available option in many locales. While PWD have long advocated remote attendance options for events and lessons [61] only now are remote options broadly available. However, the tweets we examined revealed that the quick adoption of online education tools and programs has introduced new accessibility barriers. Educators with little or no previous experience instructing remotely must make do with little training or expertise in the tools they are given. In some cases, special education programs that children with disabilities had access to at school did not translate well, or at all, to remote learning, showing that accommodations must consider emergency planning to avoid interruptions. We did see that many in the educational community, especially educators with a focus on children with disabilities, have come together online to share resources for parents to adopt and use at home. Educators also helped other educators make digital course material more accessible to address longstanding problems with inaccessible online content.

Finally, rapid access to public health information is now more important than ever. With health advice, government orders, and medical research changing at a rapid pace, it is necessary for everyone to have up-to-the-minute access to information. Public health knowledge impacts the health and safety of oneself and others, and it ensures compliance with rapidly changing social distancing policies. Yet news reports and social media posts have questioned information communication methods, particularly impacting PWD, as many governments are failing to provide accessible communication to people with vision impairments or who are deaf or hard of hearing $(\mathrm{DHH})[58,78,81]$. In our tweet analysis, Twitter users complained that press briefings and websites failed to meet basic pre-pandemic accessibility benchmarks. New and critical publichealth measures, like wearing masks in public, were implemented without consideration of their possible communication impact for 
people who are DHH. Next, we analyzed posts from the public health departments of all US states and territories, such as the example in Figure 1. We found that while the number of U.S. state health departments posting accessible images increased during the pandemic, it did so from an abysmally low baseline and limited access to public health information by people with vision impairments.

The COVID-19 crisis has emphasized the need for accessible and universal design in technology across many domains. Although the world is constantly changing, these rapid and dramatic shifts highlight gaps in strategies for emergency preparedness for PWD, a particularly understudied facet of much disaster response research in HCI. Some highlighted gaps include existing problems magnified by the pandemic, while others consist of novel emergencies that may have been ameliorated with more flexible technology and policy design. We argue that these changes should be implemented and considered before disasters occur so that marginalized groups do not feel the brunt of the impact. Moreover, we should take advantage of the current circumstances-in which nearly everyone is living a more virtual life-to create more universally accessible tools and experiences for all.

\section{RELATED WORK}

Our research lies at the intersection of social media usage, service interruptions, and public health dissemination. We examine literature pertaining to each of our domains of interest below.

\subsection{Social Media}

2.1.1 Social media and lived experiences. Social media platforms have been used to document and understand a variety of lived experiences of PWD [6, 10, 13, 36, 45, 52, 53, 65, 77]. A noteworthy area of recent research is online activism. PWD have long used social media to call attention to important issues by attempting dialogue with technology companies about tool inaccessibility [12], sharing photographs of accessibility problems with \#accessibilityfail [51], broadcasting art projects [17], and increasing disabled voter turnout [35]. Social media has also hosted large-scale events in substitute of in-person protests to avoid many of their physically, environmentally, or socially inaccessible aspects. Li et al. [50] described how PWD took to online platforms to organize and participate in the 2017 Disability March which energized, empowered, and motivated self-change by attendees. Subsequent research examined Twitter usage by the campaign against legislation which would change the Americans with Disabilities Act [5]. They found that Twitter is a convenient platform for political engagement because it is used by other key players (e.g., politicians, media, and organizations) and can be impactful in bringing attention to a cause. Given its rich potential to offer windows into the lives of disabled people without requiring synchronous contact, which is particularly difficult and potentially unethical to organize during a pandemic, we positioned Twitter as a site for learning about COVID-19's impact on PWD.

2.1.2 Social media in crises. Previous research has examined social media responses during earlier crises. Information-specific activities have been found especially prevalent during crises. However, the types of tweets shared, such as a higher frequency of broadcasttype tweets than original content differs from calm times $[44,47,79]$.
For example, people affected by the DeepWater Horizon oil spill flocked to Twitter to express uncertainty regarding interventions that were being reported by traditional media, and used the platform to corroborate and interpret different information sources in the spill's aftermath [26]. During Hurricane Sandy, Twitter users in the storm's path more often retweeted local sources with locallyactionable information [47] and increased tweeting overall [41, 48]. Demuth et al. [27] noted that cues such as the closing of local businesses prompted users to more intentionally assess risk, which resulted in the discussion of protective measures online. In addition, when surveying PWD $(n=1,120)$ about how they received the public alerts, $15-21 \%$ reported using social media to receive emergency information, $10-15 \%$ reported using social media to verify the information, and $10-18 \%$ reported sharing it [55]. People with communication disabilities and those who are deaf reported the highest use of social media during emergencies. Overall, social media has been shown to be a key information source and deliberation platform for receiving and processing rapidly-changing phenomena and for proposing and discussing potential responses.

\subsection{Service Interruptions}

Crisis events like weather phenomena and pandemics necessitate the disruption of ordinary activities, such as the closure of businesses and schools. It is therefore essential that back-up services meet the needs of people with a diverse range of abilities. In this next section, we explore literature in two domain areas that we focus on in this research.

2.2.1 Shopping online. In the event of physical store closures, online stores serve as a potential stand in for the population. This introduces shifts in inequality of access, as PWD historically have been less connected to the internet and unable to use several websites, particularly if they are older, have lower incomes, or live alone [4, 30, 32, 69]. However, online retail also has a history of biasing against PWD; access barriers on the internet are actually widespread [75]. In the U.S., a number of lawsuits have been filed to compel retailers to update their websites to meet accessibility standards, including cases against the Winn-Dixie grocery store chain [60], Domino's Pizza restaurants [14], Burger King restaurants [15], and Blue Apron meal kit delivery [20]. These and other ecommerce websites were found to be inaccessible by screen readers, making it impossible for users with vision impairments to get the products they want [12]. These access barriers can have psychological as well as practical impacts on shoppers [37]. The outcomes of these cases recommend basic accessibility features to improve the shopping experience, including captioned and audio-described videos and keyboard navigation support [54]. In addition to technological barriers, websites sometimes have online prices that are higher than in-store prices, producing pricing discrimination for people who cannot easily shop in a store [49]. As the world transitioned to this traditionally inaccessible form of shopping, we examined a sample of tweets depicting the experience and advocacy surrounding a crucial activity for survival: online grocery shopping.

2.2.2 Education online. While access to online education is convenient, the online interface presents a myriad of challenges for students and instructors with disabilities [66]. Like online shopping, 
access barriers are persistent and widespread. For example, learning management systems introduce accessibility problems, as has been extensively found in research on Massive Open Online Courses (MOOCS) $[9,18,46]$. A personal account by Professor Sushil Oswal, who is blind, recently detailed his difficulties teaching through the Canvas online learning management system [62]. In response, several research teams have written guidelines for improving the accessibility of MOOCs, including those on EdX [68], Coursera [2], and others [21, 23]. However, access barriers exceed software interaction design; screen time, for example, exacerbates eye fatigue [66]. Finally, Seale and Cooper [71] posit that learning theories can reveal an association between pedagogy and accessibility. That is, even if tools become functionally accessible, the curriculum and culture of a course impacts accessibility. One study [66] corroborated this as $69.7 \%$ of disabled students surveyed kept their disabilities private and $71 \%$ chose to not request accommodations. In light of this multifaceted problem, researchers have argued that teachers must implement universal design for learning as to not rely on disability disclosure to facilitate accessible instruction [16, 31]. In parallel, movements to widely teach web accessibility to computer science are burgeoning in hopes more professionals, like the developers who build and maintain learning management systems, will more consistently follow accessibility best practices [65, 73]. Despite these steps forward, we lack research to understand how a rapid transition to online education affects PWD.

\subsection{Public Health}

The timely dissemination and consumption of information communicating impending risk and necessary precautions during crises is crucial to decision making and even citizen safety [63]. However, planners have a track record of forgetting to account for the unique needs of persons with disabilities. Numerous court cases in the United States have been filed regarding emergency response plans that violated the Americans with Disabilities Act by omitting provisions for PWD. The court has often ruled against local and regional governments, citing a lack of procedures for notifying PWD of an emergency (Los Angeles, CA), no plans to inform PWD how to react in an emergency (Los Angeles and Sacramento, CA), and an absence of provisions to evacuate PWD during emergencies (Brooklyn, NY) [38].

Researchers have investigated the effects of such inadequate emergency planning and policies on PWD. For example, deaf evacuees were turned away from two shelters during two hurricanes on the U.S. Gulf Coast [81]. During Hurricane Katrina, persons who were deaf or hard of hearing had a difficult time communicating in shelters due to a lack of assistive devices ( $80 \%$ of shelters) and translation services (70\% of shelters) [58]. Similar findings were reported among the Deaf communities in Japan that experienced the Kobe and Tohoku earthquakes, and many members of the Deaf community elected to stay in damaged homes [78]. In addition to a lack of accessible services in emergencies, Campbell and colleagues cautioned that during pandemic influenza, PWD who rely on caregivers may be at greater risk of infection given the close-proximity human contact much caregiving requires [19]. Overall, individuals with disabilities face undue burdens and increased challenges when attempting to access public information and assistance during emergencies.

\section{METHODOLOGY}

To conduct this multi-domain, multi-phased study, we first performed a widely inclusive search to learn what PWD were tweeting about regarding COVID-19. Second, we synthesized our domains of focus based on the search results and topics appropriate for an ASSETS audience. We obtained broad viewpoints on the impact of critical service provision, remote education, and public health information dissemination by analyzing a sample of tweets related to each domain. Third, based on what the tweets surfaced, we chose a sector of each domain to investigate in more depth.

Specifically, we began by searching for hashtags on Twitter related to accessibility and/or disabilities in general (e.g., "accessibility", "disability", "\#a11y", "accessible technology", "\#captions", etc.) and to the intersection of accessibility, disabilities, and the pandemic (e.g., “\#DisabilityC19”, “\#HighRiskCovid19”). We conducted this search of 59 general terms relating to disability and technology (available in Supplemental Material) from April 6th to April 12th, 2020 and collected 3,375 resulting tweets. We read a randomized subset of this general sample, noting particular topics and key terms that returned relevant or irrelevant results. From this process, we identified a few major topics of discussion:

- Grocery delivery: A large increase in the use of grocery delivery services and applications was leading to digital crowding and other issues.

- Online education: The transition in education at all levels to remote instruction generated conversation on accessibility requirements and accommodations.

- Remote work: Similar to the transition to learning online, working virtually and at home generated conversation about what accommodations were available for PWD before and during the pandemic.

- Public health information: During the pandemic, many organizations shared information about preventing coronavirus spread and updates about the status of their area or nation. People discussed whether this information was accessible to those with disabilities.

- Medical rationing: The question of limited access to healthcare, including COVID-19 tests and treatments, drove discussion about disability rights and ensuring that people would receive equal access to healthcare if rationing occurred.

Of these topics, we elected to focus on grocery delivery, online education, and public health messaging because of their relationship to accessible technology and their prevalence in the general sample. Concerns around remote work significantly overlapped those related to online education. However, there was a higher prevalence of tweets related to online education, so we focused on that topic for a broader view into remote productivity. For medical rationing, concerns focused on disability rights and medical ethics rather than technology, so the topic was not within the scope of this project.

For each of the three chosen domains, we iteratively created lists of search keywords using the Twitter API to collect tweets, determine their relevance, and choose keywords to better target the intersection of the COVID-19 pandemic, PWD, and the domain. 


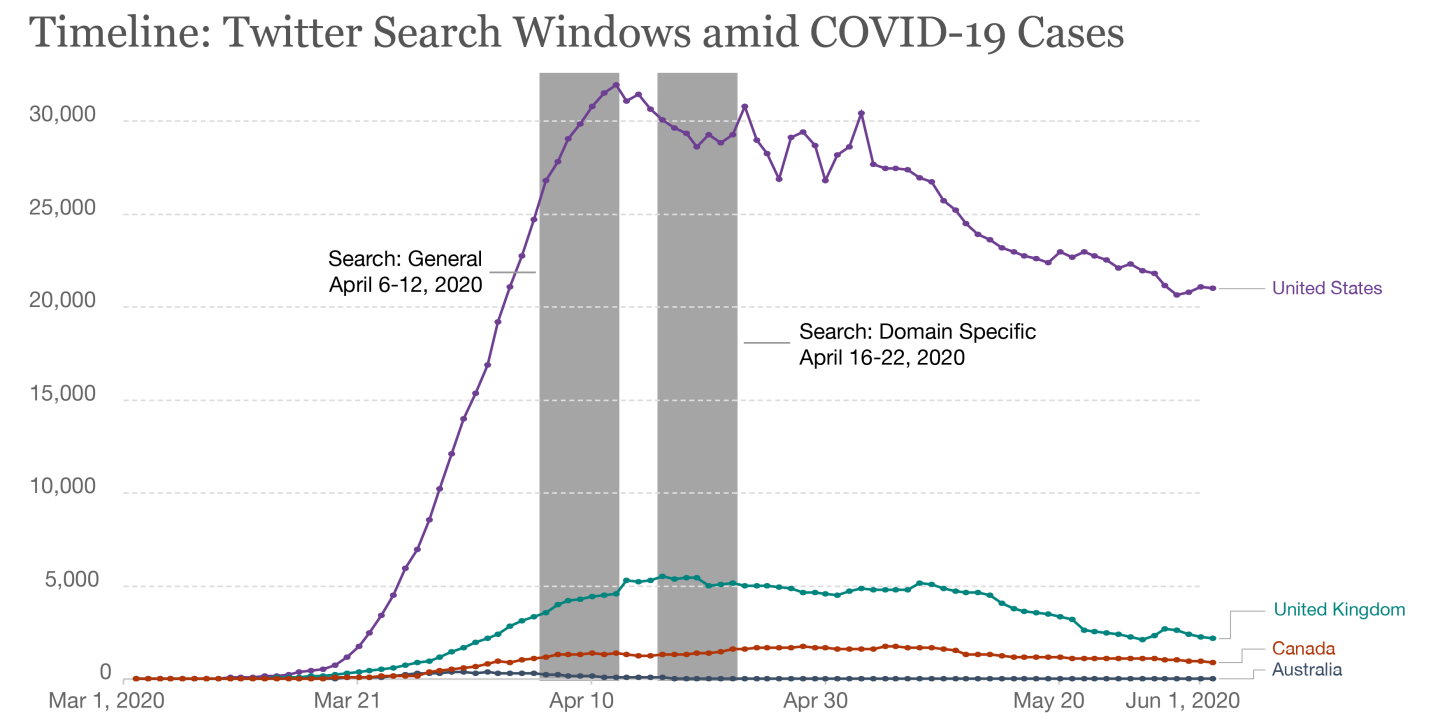

Figure 2: Timeline of our Twitter searches amid the new cases per day (7-day rolling average) in the US, UK, Canada, and Australia. Note that most countries or sub-national regions had some sort of social distancing measures, stay at home orders, or lockdowns during this time period. [CC BY] (Our World In Data https://ourworldindata.org/coronavirus-data-explorer.

We then utilized these search terms to collect a final sample of representative tweets for each domain from April 16-22, 2020 (Figure 2). We sampled the following number of tweets for each domain (no retweets were included): Grocery Delivery: 7,877; Education: 1,244; Public Health Messaging: 544 (not including the analysis of public health departments). We used the API to collect all the tweets available for each search term on each day of the selected week. Once this sample of tweets was collected, we randomly sampled 250 tweets from the total pool to analyze their themes. These 750 tweets were authored by 624 unique accounts from the following countries: $45 \%$ US, $15 \%$ UK, $5 \%$ Canada, $2 \%$ Australia, $6 \%$ other country, and $27 \%$ unknown (location is not a required profile field). Therefore, our findings present primarily a US perspective, not that of all English-speaking countries. We further calculated user activity for the accounts in our sample of tweets: $18 \%$ of users posted more than 1,000 tweets in the last 6 months, $46 \%$ between 100 and 1,000, 29\% between 10 and 100, and 7\% less than 10 tweets.

One pair of research team members analyzed the tweets for grocery delivery and education, while another pair focused on public health messaging. Members of the research team met repeatedly to discuss relevant themes in the tweet text or linked webpages. If they determined a tweet was not relevant to COVID-19, being disabled or high risk, or the specific domain, they replaced it with a new randomly sampled tweet to ensure that the same number of tweets was assessed for each domain. Both research team members analyzed the entire sample to discuss and develop themes together.

For each domain, we present the themes elicited by the tweets themselves as well as additional findings from linked news articles, resources, or additional domain-specific analyses. We aimed for a balance of coverage and specificity by iteratively expanding and refining our terms before searching, nonetheless our search did not include all possible related topics that could impact PWD, such as remote healthcare. As mentioned previously, our findings are not intended to be an exhaustive description of all impacts on PWD, but to serve as a reflection for technology design and accessibility impact for unusual or emergency situations.

\section{FINDINGS: CONTACT-FREE GROCERY ACQUISITION}

In this section, we examined the degree to which distribution of critical services prioritized PWD and high-risk individuals. We scoped this domain to the critical service, grocery acquisition, given that it is a necessary activity to fulfill basic human needs. Though underresearched, nourishment has been argued a worthwhile design space (e.g., $[24,70])$ : technology could support more sustainable food production and consumption [59] and may help address undernourishment in food deserts, which are home to many PWD [29]. Overall, food acquisition by PWD is very under-studied.

Our analysis consisted of two phases. In the first phase, we learned what tweets revealed about the grocery acquisition landscape, given our preliminary findings from the media and initial tweet samples on the prevalence of negative experiences and ambiguity about grocery acquisition by high-risk and disabled people. In the second phase, we performed a follow-up exploration of the COVID-19 policies of large, relevant supermarket chains to corroborate the tweeters' reports.

\subsection{What do tweets reveal about grocery acquisition?}

As we began our Twitter exploration, our sample revealed four primary themes. The first two centered on product delivery, an accessibility hack that disabled people have leveraged for years as online shopping may make product perusal more accessible, require 
less energy, and necessitate fewer movements than a trip to the store. In the first theme, tweeters were grateful for mutual aid efforts and for any provisions that supermarkets made to allocate delivery slots for people who could not shop in person. However, the second theme highlighted insufficiencies in these special provisions, such as PWD's inability to secure grocery slots when services were flooded with requests. Notably, experience sharing about grocery acquisition superseded delivery concerns. Specifically, a third theme surfaced in a multitude of requests for help and reports of health and disabilities that made obtaining food especially difficult. Finally, some tweeters exhibited a fourth theme of public advocacy in the form of questioning governments and supermarkets and sharing their preferences for how grocery acquisition should be handled during COVID-19.

In our first theme, tweeters shared positive responses to food insecurity during COVID-19. Some praised philanthropists and localized mutual aid efforts for serving those in need. Others shared information about how those in need of groceries could connect with mutual aid in their area. A third subset represented personal accounts of high-risk and disabled people's experiences receiving groceries as part of government and corporate efforts to serve them equitably. For example, one tweeter claimed supermarket chain Iceland Groceries "saved the day" by delivering groceries to their high-risk father. Together, these tweets highlighted communities coming together in recognition that grocery shopping for high-risk and PWD warranted equitable assistance provision.

However, our second theme showed that disabled customers of grocery delivery platforms encountered new challenges. For example, use of delivery services by people who typically shop in stores spiked, leaving several disabled and high-risk customers unable to secure a delivery slot when best practices cautioned them against venturing to supermarkets. Another Iceland Groceries shopper, for example, had a different experience. They recounted being a vulnerable high-risk citizen and not being able to get grocery deliveries for the next week, "...I understood you were making delivery slots for customers like myself." Some of these negative experiences were spawned by ambiguity about how qualification criteria were developed for preferential delivery slots as part of a government-stipulated, supermarket-run program in the UK. Most acknowledged they did not officially qualify but believed the qualifications too narrow as to not include caregivers or some disabilities. As one tweeter summarized, "Despite being in the high-risk group, we're not in the highest risk group." These snippets hinted at some complexities of changing policies: while helping some individuals, they left out others.

The third theme encapsulated the sharing of challenges that made grocery acquisition difficult and their associated requests for help. Some of these posts directly responded to philanthropists whose offers to assist random tweeters went viral. Others contributed to more general conversations, such as educating the Twitterverse of specifics about health risks and caring for loved ones. One tweet sought help and justified the request with a description of their family's challenges, "please help my mom dad sister and I are all out of work due to being high risk Need help with bills and groceries." This theme demonstrated that there are grocery-related needs beyond delivery slots: many tweeters lacked funds or other structural supports that would make grocery shopping easier.
In the fourth theme, Twitter was used as a platform for public advocacy $[12,50]$. Some tweeters tagged government officials and supermarkets with questions. For example, a high-risk customer was disconcerted that they had purchased a membership but could not secure delivery, "I've been a weekly customer for years Now I can't get a delivery spot ... You dont [sic] pick up the phone or allow email How should I handle this Reverse membership fee." Another concerned tweeter tagged a provincial government asking, "Is there anything that the [anonymous] government is doing to help those on [government assistance] ... They live on very small amounts of money and with rising food pharmaceuticals and delivery costs they aren't making it through." Other advocacy took many forms: critiques that current service provisions were insufficient (19 tweets), advice to individuals on how to cope and be helpful to others, messages to governments and private companies with policy suggestions, general education (as opposed to personal stories) about what it is like to be high-risk or disabled during COVID-19, and calls for volunteers to grocery shop for neighbors. For example, one critique argued online shopping became more difficult for PWD: "Disabled people who couldn't shop STILL can't shop but most have lost access to online delivery" because many nondisabled people newly used grocery delivery services with the onset of COVID-19. Some suggestions were practical advice: "Take care of your high-risk friends and family Drop groceries off on their porch assist financially if you are able Just love them."

Conversations on Twitter about grocery acquisition while being high-risk or disabled spanned a variety of themes from positive to negative sentiments, personal accounts and activist stances. Although mutual aid, government policies, and changes in customer service to equitably assist disabled and high-risk people were well received by some, there remained significant need for additional provisions and clarity on how to negotiate them.

\subsection{What are grocery store reactions to COVID-19?}

To gain an understanding of the context of the tweets that we examined, we surveyed the policies of all of the grocery store chains with over 1,000 stores in the US and UK, the primary tweet locations in our sample: Kroger, Albertson's, Walmart, Target, Food Lion, Tesco, and Sainsbury's (Table 1). For each company, we searched for information regarding policies that could impact the accessibility of acquiring groceries based on our observations from the tweets. This information included changes to the in-store shopping experience in light of COVID-19 (e.g., capacity, limitations to instore movement, availability of priority hours) and delivery/pickup accommodations for customers at high risk and/or with disabilities. While in-store policies may not directly relate to delivery services, store conditions (e.g., long outdoor queues) may lead to additional users requiring delivery. For each interest area and category, we examined each grocer's COVID-19 response webpage and searched for press releases or other media if the information was not available there. This information is presented in Table 1 . If the corporate website did not explicitly mention information about an interest area, we excluded the topic from Table 1, and we stated "by store" if the corporate messaging reported that the policy varied by individual store location. 
Table 1: For large grocery store chains $(1000+$ stores) in the US and the UK, we analyzed policies related to the accessibility of obtaining groceries. For each store, we detail changes to in-store shopping including capacity limits (Capacity), in-store flow (In-store), priority shopping hours (Priority hours), Delivery, Pickup, Priority Delivery, Priority Pickup, and Limits (e.g., if the store mentioned delays that may limit people from delivery access, or if they placed restrictions on slots that users could sign up for). Abbreviations: CF for contact-free. *Target is followed by an asterisk to indicate that Target paused their rollout of grocery delivery services due to increased in-store demand and limited trained staff. ${ }^{* *}$ Two asterisks indicate the stores are multinational companies for which we examined their US-specific or UK-specific policies only. This data was collected on April $23^{\text {rd }}, 2020$ and verified again on May $5^{\text {th }}, 2020$.

\begin{tabular}{|c|c|c|c|c|c|c|c|}
\hline & Kroger (US) & Albertson's (US) & $\begin{array}{l}\text { Walmart } \\
\left(\text { US }^{* *}\right)\end{array}$ & Target $^{*}\left(\mathrm{US}^{* *}\right)$ & $\begin{array}{l}\text { Food Lion } \\
\text { (US) }\end{array}$ & Tesco $\left(\mathrm{UK}^{* *}\right)$ & $\begin{array}{l}\text { Sainsbury's } \\
\text { (UK) }\end{array}$ \\
\hline Capacity & $50 \%$ & - & $20 \%$ & by store & - & by store & by store \\
\hline In-store & $\begin{array}{l}\text { floor } \\
\text { markers }\end{array}$ & floor markers & $\begin{array}{l}\text { floor markers, } \\
\text { more entrances }\end{array}$ & floor markers & $\begin{array}{l}\text { large carts } \\
\text { only }\end{array}$ & floor markers & floor markers \\
\hline Priority hours & $\begin{array}{l}2 \mathrm{hrs} / \mathrm{d} \\
\text { at-risk }\end{array}$ & $\begin{array}{l}\text { by store, } \\
\text { at-risk }\end{array}$ & $1 \mathrm{hr} / \mathrm{wk}, 60 \mathrm{yrs}+$ & $\begin{array}{l}2 \mathrm{hrs} / \mathrm{wk} \\
\text { at-risk }\end{array}$ & $\begin{array}{l}2 \mathrm{hrs} / \mathrm{wk} \\
\text { at-risk }\end{array}$ & $\begin{array}{l}3 \mathrm{hrs} / \mathrm{wk} \text {, at } \\
\text { risk }\end{array}$ & $\begin{array}{l}3 \mathrm{hrs} / \mathrm{wk} \text {, at } \\
\text { risk }\end{array}$ \\
\hline Delivery (D) & $\mathrm{CF}$ & in home, $\mathrm{CF}$ & $\mathrm{CF}$ & $\begin{array}{l}\mathrm{CF} \text {, paused } \\
\text { groceries }\end{array}$ & $\mathrm{CF}$ & in home, CF & $\mathrm{CF}$ \\
\hline Pickup (P) & distancing & $\mathrm{CF}$ & $\mathrm{CF}$ & $\begin{array}{l}\mathrm{CF} \text {, paused } \\
\text { groceries }\end{array}$ & $\mathrm{CF}$ & by store & $\begin{array}{l}\text { collection } \\
\text { point }\end{array}$ \\
\hline Priority D & - & - & - & - & - & $\begin{array}{l}\text { govt. lists, case } \\
\text { by case }\end{array}$ & $\begin{array}{l}\text { govt. lists, } \\
\text { existing info }\end{array}$ \\
\hline Priority $\mathrm{P}$ & - & - & $1 \mathrm{hr} / \mathrm{d}$, at risk & - & - & - & - \\
\hline Limits & - & delays & delays & delays & delays & slots & slots \\
\hline
\end{tabular}

All of the grocery store websites referenced physical changes to in-store shopping, such as reductions in customer capacity, floor markings to specify travel direction and inform where people may stand, and the elimination of small carts and baskets. All stores also specified priority hours for shoppers either who were "at risk" (including terms such as at risk, seniors, vulnerable, pregnant, and immunocompromised or references to CDC guidelines) or, in the case of Walmart, only seniors. While Walmart did not offer highrisk shoppers' priority in-store hours, Walmart was the only US store with priority pick-up hours and they let people self-enroll as high-risk online. All stores offered contact-free delivery, and most supermarkets also offered contact-free pickup (via placing groceries in the trunk of a car). Albertson's and Tesco mentioned that delivery people could deliver groceries and place them inside of the home. All stores except Kroger acknowledged that people should expect delivery delays. Tesco and Sainsbury's additionally enrolled people in priority grocery delivery slots by consulting government-curated lists of extremely vulnerable people, prior customer information, and case-by-case requests, and they limited the number of slots that non-priority customers could access.

Overall, official policies around prioritizing grocery deliveries for people who needed it the most were vague or non-existent, even while companies acknowledged delays and lack of delivery slots. Our Twitter analysis revealed that without specific measures to support customer prioritization with increased demands, individual customers for whom in-store shopping is a challenge must navigate grocery shopping by contacting companies individually (e.g., by tagging companies or government officials in tweets) or by leveraging their social support systems. While Tesco and Sainsbury's (UK chains) offered prioritization, they also placed a notice on their sites encouraging customers to limit their use of delivery services such that people who need delivery can access the service. Given that messaging during the public health crisis can be confusing to the general public, clear guidelines educating people to save delivery options for those who need it may encourage some to shop in-store.

\section{FINDINGS: SOCIALLY DISTANT EDUCATION}

In many areas, social distancing started with the closure of schools from elementary through post-secondary education. We examined the transition to remote education for disabled students, their instructors, and caregivers during the initial months of social distancing through themes from our Twitter sample. The most notable themes include: supporting social and emotional learning and wellbeing, advocacy and critiques for available education accommodations, and resource sharing. Given the amount of resource sharing about access technology and how to make educational content accessible, we further categorized the tools and tips named in our Twitter sample and discussed the challenges of moving education to a fully online format during this transition.

\subsection{Supporting social emotional learning and wellbeing}

Approximately $20 \%$ of analyzed tweets discussed social emotional resources for education at home. Social and emotional learning (SEL) has emerged as an important pedagogical tool to facilitate 
academic success and mental wellbeing (e.g., [22, 33, 74]). SEL approaches include self-management, self-awareness, and recognizing emotions in others [22, 34]. To assist in the transition to online education, instructors and therapists shared resources that aimed to facilitate emotional regulation and to encourage students to meet their basic needs first and to not over-worry about completing coursework in the same way as they did pre-pandemic.

The most commonly shared resources covered DIY crafts, methods for scaffolding healthy routines, and how to provide sensory support for children. These included guides on how to create quiet spaces in the home and calming stimulation. Some resources showed how to create visual routines and schedules to parents of students with autism. Another subset shared literacy resources to support speech therapy at home and to facilitate learning through play. Finally, other tweets from therapists and special educators concerned navigating learning new technology to adapt their workflow, check-in with their students, and support parents and caregivers.

\subsection{Advocacy and critiques of available education accommodations}

Students with disabilities shared personal challenges on Twitter, including struggles to complete tasks at home due to chronic health conditions. Others offered strong critiques against universities who ignored their accommodation requirements before the pandemic, but now were able to make those accommodations for everyone [61]. Tweets mentioned that pre-pandemic accessibility accommodations were assessed on a "special-needs" basis instead of using accessibility as a best practice overall. A specific tweet lamented that despite online coursework having both positive and negative outcomes for broader accessibility, the current need basis approach to accessibility prevented schools and universities from being better prepared for the current situation.

Tweets (12) also surfaced problems with instructors not having enough training on accessibility, resulting in ableist solutions. For example, one tweet discussed ableist proctoring practices that were implemented in remote testing procedures such as using "irregular" eye-movement as a sign of possible cheating.

Advocacy-related tweets urged parents and students to prioritize flexibility amidst the pandemic, but to also assert their continued right to education. Some tweets in this theme took political stances, urging legislators to not approve any waivers that would excuse educators from ensuring at-home instruction included disabled students to the greatest degree possible. This was sparked by a legislator-sponsored proposal to relax requirements stipulated by the US's Individuals with Disabilities Education Act [1]; the proposal was ultimately not adopted [72].

\subsection{Sharing resources on accessible educational technologies}

We observed a high percentage of tweets $(\sim 25 \%)$ sharing resources on how to make online content accessible for students with disabilities. The resources and tips shared were directed towards teachers, students, and parents; they span across four categories: general accessibility features, educational content creation, content consumption, and live conferencing support.
Resources shared related to general accessibility features include features of various devices (e.g., Macs, Windows PCs, Chromebooks, iPads) such as screen readers, magnifiers, contrast adjustments, dictation, and narration, though these are not specific to education settings. For content creation, many tweets shared resources to suggest that teachers make course content easy to parse, add alt text to images, and generate captions for videos automatically (e.g., using techniques like Flipgrid, Screencastify or Apple Clips) or manually (post-hoc editing). Also, many specific features were suggested to students and parents to assist with their content creation, such as using dictation for voice typing and changing to full screen to reduce distractions. For content consumption, many tweets focused on tips for students to better perceive the educational materials. Resources included display support (e.g., zooming features in word processing software), cognitive support (e.g., Microsoft immersive reader), and translation (e.g., Google Translate). Many companies and organizations advertised available and accessible educational content that were specifically prepared for students with disabilities.

Because most traditional face-to-face classes moved to online formats, people heavily discussed live conferencing platforms and support, including Zoom, Google Hangouts, Skype, Microsoft Teams, and Webex. The resources shared focused on whether the platforms are screen reader accessible, ways to support Q\&A, whether participants can join in using alternative methods (e.g., dial-in number), and whether and how the tools can support live captioning. The highest praised methods included automatic captioning support by Google Slides and Microsoft PowerPoint or integrating external captionists or plugins (e.g., Otter.ai) for Zoom and Webex.

When moving education online, people pieced together solutions from various platforms to adapt to accessibility barriers. In the long run, many of these problems require technical solutions from companies to improve the accessibility features. However, some problems arise from a lack of knowledge and awareness of existing technical capabilities by the end users. Therefore, the broader sociotechnical context should also be considered to make sure existing solutions can be utilized effectively.

\section{FINDINGS: PUBLIC HEALTH MESSAGING}

In a crisis, access to up-to-date information is critical to ensure collective public safety. As such, our themes in this domain are concerned with the degree to which disabled people could interpret pandemic status updates and associated governmental responses. This was discussed in the contexts of broadcasted press briefings and online information.

\subsection{Communication from Governments to People}

Many of the tweets that we examined discussed the presence of sign language interpreters at government-hosted press conferences about the pandemic. Users tweeted praise directed at press conferences that included interpreters, and gratitude toward the interpreters themselves. Some hearing tweeters used this opportunity to ask their questions about sign languages, and some seemed surprised that facial expressions were important to the language. 
Discussion, primarily by advocates of Deaf and disabled communities, proliferated in critique of governments who did not have interpreters at their press conferences, citing previous violations to indicate this was an ongoing problem [19,81]. As we examined English language tweets, these criticisms were primarily leveled at the US White House and UK Prime Minister's briefings. Twitter users pointed out that many municipal, county, or state governments provided sign language interpreters, yet the US and UK national governments failed to do so. Some tweets discussed the inaccessibility of public health information online or on social media. An example in Figure 1 depicts a tweet from the CDC with a popular graphic about "flattening the curve". While versions of this graphic and accompanying slogan were ubiquitous public health messages in March 2020, the graphics were not accompanied by alternative text to describe them for people with vision impairments. As such, we further discuss the inaccessibility of government websites and social media in Section 6.3.

\subsection{Communication Difficulties for PWD}

A secondary theme that emerged from our sample was the effects of the public health recommendations to wear masks on people who are $\mathrm{DHH}$ or who have communication disabilities. We noticed that the discussions were different depending on whether they concerned communication in everyday or medical settings.

6.2.1 Everyday Communication. People who are DHH often rely on their conversation partners' faces to be visible as gestures and expressions are integral to sign languages. Additionally, interpreting aural speech necessitates face visibility as lip-reading aids in understanding verbal speech and inferring meaning [76]. Public health recommendations instructing people to wear face-obstructing PPE (e.g., face masks) rendered interpersonal communication impossible for many DHH people, excluding them from society. Many echoed the sentiment of one Deaf Twitter user, who posted "I'm in a[sic] BIG trouble. What if we are required to wear a face mask in public?... I can't lipread when his/her [sic] mouth is covered. I guess I just wave and move on..." Thus, with store employees, essential workers, and in some cases TV reporters wearing face masks, DHH people face additional challenges in accessing basic needs. Several tweets raised awareness of this problem and advised people to carry alternate communication materials (e.g., pen and paper, speech-to-text translation apps) when wearing face masks. There were also multiple tweets linking to transparent face masks to facilitate DHH communication; however, sources for their purchase were unclear. Regardless, the effectiveness of these solutions is limited by the low awareness of this problem and the even lower number of people willing to circumvent it by proactively keeping alternative communication mediums on hand. Without mention in official public health guidelines, it is unlikely that these solutions will be adopted widely enough to benefit $\mathrm{DHH}$ people in everyday communication.

6.2.2 Medical Communication. A more urgent challenge for DHH and other disabled people is the need for effective communication in medical and healthcare environments. It is even more critical for healthcare professionals to wear PPE in medical settings where contagious diseases such as COVID-19 may be present. The information conveyed between patients and healthcare workers must be communicated accurately and effectively $[25,80]$. Twitter users suggested possible solutions using technology (whiteboards, clear face masks, or remote interpreters). At least one tweet linked to a printable form that $\mathrm{DHH}$ or people with communication disabilities could complete before talking to medical professionals. Two tweets, including one by advocacy group NCIHC [57], commended specific healthcare providers for ensuring that their patients had access to in-person or remote interpreters onsite or at testing sites. In an encouraging finding, we learned that several third-party remote sign language interpreters and real-time captionists offered their services for free to facilitate medical communication. However, ideally hospitals and public health institutions would already have the necessary tools to communicate and properly treat PWD.

\subsection{Accessibility of US State Health Department Twitter Accounts and COVID-19 Websites}

While analyzing tweets regarding public health information, we noticed many government agencies attempting to disseminate information through website links, videos, or included images. As prior work has demonstrated $[39,40,56]$, we hypothesized that social media channels may still be inaccessible to people with vision impairments. To investigate this, we collected pre-pandemic tweets from the official Twitter accounts of the US Centers for Disease Control, US Health and Human Services, and all health agencies for the 50 US states, Washington D.C., and US territories. We then examined the use of alternative text descriptions for images posted by these accounts. The images on these accounts included simple eye-catching graphics about daily briefings, public health information in graphics, and various state or partner resources (Figure 3). Overall, 8 of 55 agencies' Twitter accounts had used alternative text prior to the pandemic, with 589 of $926(63 \%)$ of the images those accounts posted in November 2019 - January 2020 containing alternative text descriptions. Once the pandemic began in earnest (February - April 2020), a total of 12 accounts employed alternative text descriptions, with 1,744 of 3,111 (56\%) of their images being made accessible. While the percent of total images that were accessible dropped slightly, four new states adopted the alternative text feature on Twitter, and most states stayed close to the percentage of accessible alt text they offered prior to the pandemic. We did not examine the quality of the alt text included by states that choose to do so, although prior efforts to assess alt text quality on Twitter indicate that most human-authored alt text either partially or fully describes the image [39]. The majority of images tweeted by the states were inaccessible, and the quantity of public health inaccessibility was striking.

This lack of accessibility for pandemic updates and resources was not limited to social media. One article shared several times in our tweet sample was an analysis of the accessibility of US state COVID-19 websites [8]. Government websites must comply with standard accessibility guidelines, but this analysis found that all but 2 (Maine and New Mexico) featured common accessibility issues. For example, this study found that $41 / 50$ websites had lowcontrast text, $31 / 50$ had empty links or buttons making navigation 

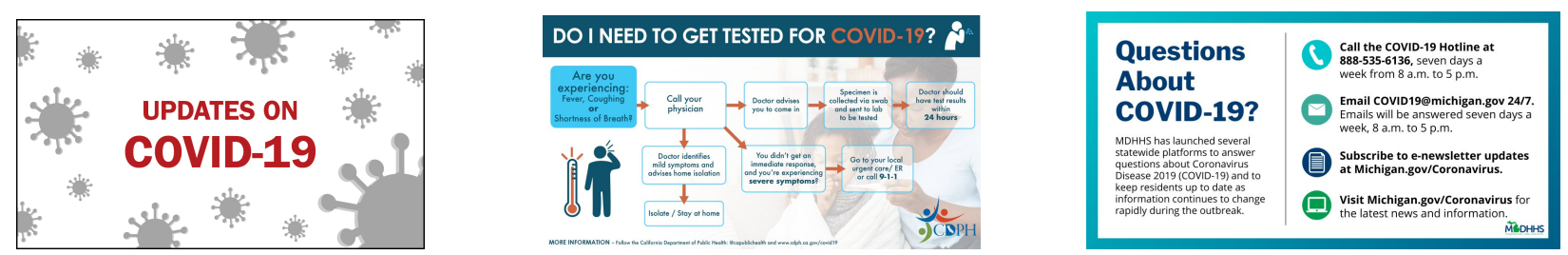

Figure 3: Examples of three common image types posted by state health departments on Twitter: (left) a graphic to catch attention and advertise daily updates posted by Massachusetts, (center) a flowchart informing people who should be tested for COVID-19 posted by California, and (right) resources for people with questions about COVID-19 posted by Michigan.

challenging, and 12/50 were missing alternative text for images. In response to this article, several states have claimed they take accessibility seriously and will work to improve their websites, albeit weeks after they were first published.

\section{DISCUSSION}

The themes which emerged in each of our three domains generally fit four different types of issues relating to emergency preparedness and accessibility (Table 2). In many ways, when institutions rapidly change or provide new guidelines (e.g., masking policies), the impacts on PWD are considered unintended consequences. However unintended, they can still impose undue burden, and may be avoidable with more inclusive prior planning. As such, technology and policy designers must always consider their effects on PWD: at the time of design, during proactive emergency preparedness and policymaking, and during an emergency itself. Failure to do so will only result in harm caused by problems that could have been foreseen and prevented. As such, our discussion offers some key questions that may guide future and much needed research on the role technology can play in emergency preparedness along with some design recommendations to improve access supports.

\subsection{Pandemic-Influence on (Re)Structured (In)Accessibility}

The rapid changes have exacerbated existing problems that have negatively affected the disability community for years, and as with prior research, we noticed Twitter became a platform for sharing associated frustrations and suggestions [5, 51]. Inaccessible websites and social media accounts are not new problems $[7,39,42,43,51,56]$, but when they contain critical public health information, they become even more important. Online education tools have always had accessibility barriers, and PWD could not effectively use them before the pandemic $[9,18,46,62]$, yet at the pandemic's onset, they became a requirement rather than an option, comprising the only gateways to resuming lessons. It is hard to create and validate accessible technology and policy during an emergency like a pandemic, so efforts must be enacted at the time of design and when things are in normal operation.

In some cases, PWD had important services or accommodations pre-pandemic that have been negatively affected or removed entirely due to social distancing policies. For example, when people who always rely on grocery delivery to meet basic needs cannot utilize the service due to crowding, the impact is compounded

Table 2: Summary of common themes across the three domains (Product Delivery, Online Education, and Public Health Communication), including existing problems being magnified by the pandemic, existing solutions don't have emergency substitutes, emergency measures did not consider accessibility, and some improvements emerged but took a pandemic to get there.

\begin{tabular}{llll}
\hline & Product Delivery & Online Education & Public Health Comm \\
\hline $\begin{array}{l}\text { Existing problems } \\
\text { magnified by pandemic }\end{array}$ & $\begin{array}{l}\text { Lack of funds and support of } \\
\text { basic needs }\end{array}$ & $\begin{array}{l}\text { Inaccessible online educational } \\
\text { content and materials }\end{array}$ & $\begin{array}{l}\text { Inaccessible websites and } \\
\text { social media messaging }\end{array}$
\end{tabular}

Existing solutions no emergency substitutes

Emergency measures not considering accessibility

Emerged improvements took a pandemic to get there
Delivery slot unavailable \& delayed for high-risk population

Ad-hoc signs and markers not accessible, long lines cause fatigue

Communities are connecting in forms of mutual aids
Educational plans and

N/A individualized programs built for in-person interactions

Online LMS/video delivery platforms introduced accessibility challenges

Accommodations previously rejected by universities are now allowed
Masking policy leads to comm challenges for people who are $\mathrm{DHH}$

Some sign language at briefings; 4 states adopt alt text on social media 
since the service was already an essential tool to gain access. In these cases, technology and policy designers (even at the retailer level), must consider how to ensure those people's needs are met. Some guiding questions to mobilize this important research include: if there are accommodations, such as captioning, in the physical classroom, how could that move online if in-person courses are disrupted? Similarly, how can we support individualized education plans and provide the same level of accommodation to students who benefitted from in-person support? What other disasters or environments may affect the planned technology or accommodation, and how can we mitigate the impacts on transition? When we design accessible technology and equitable policy for the future, we must not only consider how new experiences will be accessible, but how new experiences will successfully transfer existing accessibility solutions.

Of course, some policy or technology changes cannot be easily anticipated, as this is the nature of emergencies. But unlike current practice, emergency measures must keep people with disabilities in mind when enacted! For example, when retailors had to modify their stores to obey social distancing policies, they did not consider that ad-hoc signs or markers would be inaccessible to people with vision impairments. Similarly, the rapid public policy switch which standardized mask-wearing lead to communication barriers for people who were DHH, not only in the healthcare system, but in public, similar to past emergency response inadequacies [58, 81]. These policies must be enacted for public health and safety, but should come with guidelines for how to mitigate negative consequences for PWD, as well as allowing some flexibility in implementation where it is safe. Failure to do so will only ensure more burden falls on the people already adversely affected by an emergency.

Finally, some tweets and media reports hinted at how the pandemic may improve accommodations for PWD. For example, pandemic-inspired policies requiring that learning and working happen at home when at all possible are finally recognizing PWD who were previously denied such accommodations. Daily press briefings from various governments may have raised awareness about the importance of sign language interpretation, and we see a few state health departments may have turned on accessibility features for social media accounts that they were not using previously. We hope that some societal changes turn out to be good for PWD, but it remains to be seen whether these accommodations will remain after the emergency has passed.

\subsection{Flexibility to Support Shifting Eligibility Requirements for Services}

The assumptions that we build into our organizations and technology, such as first-come-first-serve prioritization of grocery delivery slots, may be difficult to change in an emergency, but if that code or policy was flexible, then prioritization efforts could be instituted sooner. When something can be universally designed to serve everyone better, that is the preferred approach, but if finite grocery delivery slots must be allocated, for example, equitable solutions must be pre-planned to ease their enactment. Clear-cut solutions seem impossible, but we argue that neglecting to thoughtfully design potentially tiered deployment of particular services to particular people cannot be ignored or we are complicit in recementing ableist status quos when disaster strikes. One area that our tweets taught us needs targeted attention is how to design equitable resource allocation inclusive of those who do not make it onto centralized databases of PWD like the caregivers and high-risk people who struggled to obtain groceries, even when policies were implemented to assist. However, while more equitable technology could be designed to allocate service provision based on more flexible eligibility requirements, we must also consider the ethics of such information collection. For example, research questions could explore how to embrace self-reports inclusive of people who do not have documented disabilities while minimizing taking advantage. Future work could help governments and large companies transparently communicate decisions about who is eligible and offer alternative avenues for qualifying, as Walmart allowed high-risk people to manually enroll in priority pick up. Finally, platforms must build in privacy to prevent exploitation of vulnerable information sharing to, for example, avoid selective promotion of price gouged products as online shopping is already criticized for being more expensive [49]. Additionally, improvements may come from directions which do not rely solely on those in most need providing information to justify equitable treatment. As some tweets suggested, we find promise in mutual aid models of support which accessibility research could help to scale and which may localize centers for matching people and companies who can help with those in need without requiring detailed documentation of disclosure. Further, as social media has become a tool of education, we believe retailors' platforms could also build in moments for customers to consider their need in the milieu of others' during pandemic times, with alternatives for how they may shop differently to decrease their impact on overburdened systems.

\subsection{Flexibility to Support Accessible Rapid Transitions Online}

While not all emergency measures can be planned for ahead of time, many can be anticipated or flexible enough to support rapid transitions from a physical to online medium. The transition to remote learning that we are currently observing could have been an accessible one, if the tools and content had been made accessible from the beginning. As described by Seale and Cooper 10 years ago, the culture around making curriculum accessible impacts accessibility itself [71]. A shift from the current model of accessibility that relies on PWD requesting and justifying their accommodations to one of Universal Design for Learning-making accommodations easy to access at any point in time by all students, could better prepare institutions for remote teaching and learning and minimize the amount of disability-specific changes during a rapid transition $[16,31]$. Some aspects of amplifying Universal Design for Learning where accessibility research may be poised to intervene include more accessible and smooth documentation of in-person, low-tech learning to cloud storage and vice versa. Finally, we found that SEL resources were among those most commonly shared. While a pandemic increases the need for SEL resources, supporting student wellbeing and mental health is gaining traction more generally $[22,34]$. Yet much research on education accessibility concerns 
the minutia of accessing educational materials, completing assignments, and participating in class. COVID-19's impact on PWD has highlighted the need for Universal Design for Learning to also concern insuring SEL is widely available offline and online, and integrated as part of an overall inclusive learning environment.

\subsection{Limitations \& Future Work}

As the COVID-19 pandemic began to affect people and organizations in March-April 2020, we rapidly collected data from social media to capture the ways in which it was affecting PWD. We chose Twitter as a source for our social media analysis because public tweets can be quickly captured and analyzed. However, we could not validate that the tweets we analyzed came only from PWD, as disability status is not always advertised on social media profiles. Additionally, we chose domains that had existing discussion on the platform and could be easily located with keyword searches, which may have skewed our analysis towards topics that were highly visible in the public eye, such as sign language interpreters on daily TV briefings. Views and issues that PWD face may not appear in this sample if that population is largely excluded from Twitter (such as young students with disabilities), or it covers a topic that people would not be comfortable publicly broadcasting. Future work could look for these additional domain topics, and engage with them individually in depth, as each contains a multitude of design opportunities for improvement.

We also believe there is opportunity for researchers to study the long-term effects of the COVID-19 pandemic, not just the rapid shift that occurred in Spring 2020. What aspects of accessibility improve over time as social distancing policies relax? Do any accommodations that were made for remote education or work continue past the height of the emergency? And what steps do technology or policy designers take to prepare for the next disaster, either explicitly or by ensuring equitable design as part of the general design of their products or policies?

\section{CONCLUSION}

This analysis of Twitter activity surrounding the COVID-19 pandemic and disability has revealed technology and structures that are too fragile to handle rapid change, leading to further inequities for PWD. Grocery stores are not prepared to prioritize delivery of basic goods to those unable to visit in person. The tools we build for learning online do not offer the same accommodations that we have so carefully designed for in-person education. And, governments are only sporadically equipped to deliver critical information to PWD. These experiences during an emergency not only confirm the common criticism in accessibility research that accessibility must be designed from the beginning, but also illuminate that to be truly equitable, we must include flexibility in technology and policy design to accommodate PWD undergoing rapid change. In the next emergency, that little-used service like grocery delivery or online and accessible education may become universally critical infrastructure, and flexibility may allow it to better serve everyone, instead of again leaving PWD behind.

\section{ACKNOWLEDGMENTS}

We thank John Robinson and Saiganesh Swaminathan for study design support and a grant from the National Institute on Disability, Independent Living and Rehabilitation Research (NIDILRR grant number 90REGE0007) for financial assistance. This material is also based upon work supported by the National Science Foundation Graduate Research Fellowship under Grant No. (\#DGE-1745016). Any opinion, findings, and conclusions or recommendations expressed in this material are those of the authors and do not necessarily reflect the views of the National Science Foundation.

\section{REFERENCES}

[1] About IDEA: https://sites.ed.gov/idea/about-idea/. Accessed: 2020-05-07.

[2] Al-Mouh Najd A.and Al-Khalifa, A.S. and A.-K.H.S. 2014. A First Look into MOOCs Accessibility. Computers Helping People with Special Needs (Cham, 2014), 145-152.

[3] Alliance, G.D. 2020. GDA's Covid-Resilience Engagement and Response.

[4] Anderson, M. and Perrin, A. 2017. Disabled Americans are less likely to use technology. Pew Research.

[5] Auxier, B.E., Buntain, C.L., Jaeger, P., Golbeck, J. and Kacorri, H. 2019. \#Handsoffmyada: A twitter response to the ada education and reform act. Conference on Human Factors in Computing Systems - Proceedings. 620, (2019), 1-12. DOI:https://doi.org/10.1145/3290605.3300757.

[6] Balani, S. and De Choudhury, M. 2015. Detecting and characterizing mental health related self-disclosure in social media. Conference on Human Factors in Computing Systems - Proceedings. 18, (2015), 1373-1378. DOI:https://doi.org/10. 1145/2702613.2732733.

[7] Bigham, J.P., Kaminsky, R.S., Ladner, R.E., Danielsson, O.M. and Hempton, G.L. 2006. WebInSight: Making Web Images Accessible.

[8] Blind Users Struggle with State Coronavirus Websites: 2020. https://themarkup. org/2020/04/21/blind-users-struggle-with-state-coronavirus-websites. Accessed: 2020-05-07.

[9] Bohnsack, M. and Puhl, S. 2014. LNCS 8547 - Accessibility of MOOCs

[10] Borgos-Rodriguez, K., Ringland, K.E. and Piper, A.M. 2019. Myautsomefamilylife: Analyzing parents of children with developmental disabilities on YouTube. Proceedings of the ACM on Human-Computer Interaction. 3, CSCW (2019). DOI:https: //doi.org/10.1145/3359196.

[11] Boyle, M. 2020. 'It's Just Like Ticketmaster': Playing the Delivery Slot Lottery During Covid-19. Bloomberg Business.

[12] Brady, E. and Bigham, J.P. 2014. How companies engage customers around accessibility on social media. ASSETS14 - Proceedings of the 16th International ACM SIGACCESS Conference on Computers and Accessibility. (2014), 51-58. DOI:https://doi.org/10.1145/2661334.2661355.

[13] Brewer, R. and Piper, A.M. 2016. "Tell it like it really is": A case of online content creation and sharing among older adult bloggers. Conference on Human Factors in Computing Systems - Proceedings. (2016), 5529-5542. DOI:https://doi.org/10. $1145 / 2858036.2858379$.

[14] Brown, K. 2019. Court Says Domino's Pizza Website Must Be Accessible to the Blind. Courthouse News Service.

[15] Bucher, A. 2018. Burger King Class Action Says Fast Food Chain's Website Violates ADA. Top Class Actions.

[16] Burgstahler, S. 2009. Universal design in higher education: Promising practices. Seattle, WA: DO-IT, University of Washington. (2009).

[17] Burke, L. and Crow, L. 2017. Bedding out: Art, activism, and Twitter. Disability and Social Media: Global perspectives (2017). (2017), 57-74.

[18] Calle-Jimenez, T., Sanchez-Gordon, S. and Luján-Mora, S. 2014. Web accessibility evaluation of massive open online courses on Geographical Information Systems. IEEE Global Engineering Education Conference, EDUCON (Jul. 2014), 680-686.

[19] Campbell, V.A., Gilyard, J.A., Sinclair, L., Sternberg, T. and Kailes, J.I. 2009. Preparing for and responding to pandemic influenza: Implications for people with disabilities. American fournal of Public Health. 99, SUPPL. 2 (2009), 294-300. DOI:https://doi.org/10.2105/AJPH.2009.162677.

[20] Cave, B. 2017. Online Retailers Beware: Court Holds Website Violates ADA Despite Lack of Physical Store. Lexology.

[21] Chatterjee, R., Juvale, D. and Cherrez, N.J. 2017. A MOOC for U: The design of a MOOC based on Universal Design for Learning. Society for Information Technology \& Teacher Education International Conference (2017), 143-148.

[22] Cherniss, C., Extein, M., Goleman, D. and Weissberg, R.P. 2006. Emotional intelligence: what does the research really indicate? Educational psychologist. 41, 4 (2006), 239-245.

[23] Cinquin Pierre-Antoineand Guitton, P. and S.H. 2018. Towards Truly Accessible MOOCs for Persons with Cognitive Disabilities: Design and Field Assessment. Computers Helping People with Special Needs (Cham, 2018), 146-153. 
[24] Comber, R., Hee-jeong Choi, J. and Hoonhout, J. 2014. Designing for HumanFood Interaction: an introduction to the special issue on "Food and Interaction Design." International fournal of Human-Computer Studies. 72, 2 (2014), 181-184. DOI:https://doi.org//https://doi.org/10.1016/j.ijhcs.2013.09.001\}.

[25] Coronavirus poses added challenges for hospital patients who are deaf or hard of hearing: 2020. https://www.latimes.com/california/story/2020-04-16/coronavirusdeaf-hearing-hospitals-interpreters. Accessed: 2020-05-07.

[26] Dailey, D. and Starbird, K. 2015. "It's raining dispersants": Collective sensemaking of complex information in crisis contexts. Proceedings of the ACM Conference on Computer Supported Cooperative Work, CSCW. 2015-Janua, (2015), 155-158. DOI:https://doi.org/10.1145/2685553.2698995.

[27] Demuth, J.L., Morss, R.E., Palen, L., Anderson, K.M., Anderson, J., Kogan, M., Stowe, K., Bica, M., Lazrus, H., Wilhelmi, O. and Henderson, J. 2018. "Sometimes da \#beachlife ain't always da wave": Understanding People's Evolving Hurricane Risk Communication, Risk Assessments, and Responses Using Twitter Narratives. Weather, Climate, and Society. 10, 3 (2018), 537-560. DOI:https://doi.org/10.1175/ wcas-d-17-0126.1

[28] DeVault, N. 2018. Home Delivery Services Serve Up Improved Accessibility to Food and More. AmeriDisability: America's Disability Community.

[29] Dillahunt, T.R., Simioni, S. and Xu, X. 2019. Online grocery delivery services: An Opportunity to Address Food Disparities in Transportation-scarce Areas. Conference on Human Factors in Computing Systems - Proceedings. (2019), 1-15. DOI:https://doi.org/10.1145/3290605.3300879.

[30] Dobransky, K. and Hargittai, E. 2016. Unrealized potential: Exploring the digital disability divide. Poetics. 58, (2016), 18-28.

[31] Dolmage, J.T. 2017. Academic ableism: Disability and higher education. University of Michigan Press.

[32] Duplaga, M. 2017. Digital divide among people with disabilities: Analysis of data from a nationwide study for determinants of Internet use and activities performed online. PloS one. 12, 6 (2017)

[33] Elias, M. 1997. The missing piece: Making the case for greater attention to social and emotional learning in the schools. Education Week. 17, 5 (1997), 36-38.

[34] Elias, M.J. 2004. The connection between social-emotional learning and learning disabilities: Implications for intervention. Learning Disability Quarterly. 27, 1 (2004), 53-63.

[35] Ferguson, A. 2016. The \#CripTheVote Movement Is Bringing Disability Rights To The 2016 Election. Huff Post.

[36] Feuston, J.L. and Piper, A.M. 2018. Beyond the coded gaze: Analyzing expression of mental health and illness on instagram. Proceedings of the ACM on HumanComputer Interaction. 2, CSCW (2018). DOI:https://doi.org/10.1145/3274320.

[37] Fuchs, E. and Strauss, C. 2012. Online Shopping Involving Consumers with Visual Impairments-A Qualitative Study. International Conference on Computers for Handicapped Persons (2012), 378-385.

[38] George, A. and Keith, E.F. 2019. Disability Rights and Dignity in Disasters. Natural Resources \& Environment. 34, 2 (2019), 53-55.

[39] Gleason, C., Morris, M.R., Carrington, P., Kitani, K.M., Cassidy, C. and Bigham, J.P. 2019. "It's almost like they're trying to hide it": How user-provided image descriptions have failed to make twitter accessible. The Web Conference 2019 Proceedings of the World Wide Web Conference, WWW 2019 (May 2019), 549-559.

[40] Gleason, C., Pavel, A., McCamey, E., Low, C., Carrington, P., Kitani, K.M. and Bigham, J.P. 2020. Twitter A11y: A Browser Extension to Make Twitter Images Accessible. Proceedings of the 2020 CHI Conference on Human Factors in Computing Systems (2020), 1-12.

[41] Guan, X. and Chen, C. 2014. Using social media data to understand and assess disasters. Natural hazards. 74, 2 (2014), 837-850.

[42] Guinness, D., Cutrell, E. and Morris, M.R. 2018. Caption crawler: Enabling reusable alternative text descriptions using reverse image search. Proceedings of the 2018 CHI Conference on Human Factors in Computing Systems (2018), 1-11.

[43] Hackett, S., Parmanto, B. and Zeng, X. 2004. Accessibility of Internet Websites Over Time. ASSETS 2004: the Sixth International ACM SIGACCESS Conference on Computers and Accessibility (2004), 32-39.

[44] Hughes, A.L. and Palen, L. 2009. Twitter adoption and use in mass convergence and emergency events. ISCRAM 2009 - 6th International Conference on Information Systems for Crisis Response and Management: Boundary Spanning Initiatives and New Perspectives. 6, (2009), 248-260.

[45] Huh, J., Liu, L.S., Neogi, T., Inkpen, K. and Pratt, W. 2014. Health vlogs as social support for chronic illness management. ACM Transactions on Computer-Human Interaction. 21, 4 (2014). DOI:https://doi.org/10.1145/2630067.

[46] Iniesto, F. and Rodrigo, C. 2014. Accessibility assessment of MOOC platforms in Spanish: UNED COMA, COLMENIA and Miriada X. 2014 International Symposium on Computers in Education, SIIE 2014 (Jan. 2014), 169-172.

[47] Kogan, M., Palen, L. and Anderson, K.M. 2015. Think local, retweet global: Retweeting by the geographically-vulnerable during hurricane sandy. CSCW 2015 - Proceedings of the 2015 ACM International Conference on ComputerSupported Cooperative Work and Social Computing. (2015), 981-993. DOI:https: //doi.org/10.1145/2675133.2675218.

[48] Lachlan, K.A., Spence, P.R., Lin, X. and Del Greco, M. 2014. Screaming into the wind: Examining the volume and content of tweets associated with Hurricane
Sandy Communication Studies. 65, 5 (2014), 500-518.

[49] Lazar, J. et al. 2011. Potential pricing discrimination due to inaccessible web sites. Lecture Notes in Computer Science (including subseries Lecture Notes in Artificial Intelligence and Lecture Notes in Bioinformatics). 6946 LNCS, PART 1 (2011), 108-114. DOI:https://doi.org/10.1007/978-3-642-23774-4_11.

[50] Li, H., Bora, D., Salvi, S. and Brady, E. 2018. Slacktivists or activists?: Identity work in the virtual Disability March. Conference on Human Factors in Computing Systems - Proceedings. 2018-April, (2018), 1-13. DOI:https://doi.org/10.1145/ 3173574.3173799

[51] Li, H. and Brady, E. 2016. Accessibilityfail: Categorizing shared photographs of physical accessibility problems. ASSETS 2016 - Proceedings of the 18th International ACM SIGACCESS Conference on Computers and Accessibility. (2016), 277-278. DOI:https://doi.org/10.1145/2982142.2982186.

[52] Liu, L.S., Inkpen, K. and Pratt, W. 2015. "i’m not like my friends": Understanding how children with a chronic illness use technology to maintain normalcy. CSCW 2015 - Proceedings of the 2015 ACM International Conference on Computer-Supported Cooperative Work and Social Computing. (2015), 1527-1539. DOI:https://doi.org/10.1145/2675133.2675201.

[53] Manikonda, L. and De Choudhury, M. 2017. Modeling and understanding visual attributes of mental health disclosures in social media. Conference on Human Factors in Computing Systems - Proceedings. 2017-May, (2017), 170-181. DOI:https: //doi.org/10.1145/3025453.3025932.

[54] Martin, H. 2018. Lawsuits targeting business websites over ADA violations are on the rise. Los Angeles Times.

[55] Morris, J.T., Mueller, J.L. and Jones, M.L. 2014. Use of social media during public emergencies by people with disabilities. Western fournal of Emergency Medicine. 15, 5 (2014), 567-574. DOI:https://doi.org/10.5811/westjem.2014.4.21274.

[56] Morris, M.R., Zolyomi, A., Yao, C., Bahram, S., Bigham, J.P. and Kane, S.K. 2016. “ With most of it being pictures now , I rarely use it ": Understanding Twitter 's Evolving Accessibility to Blind Users. (2016), 5506-5516.

[57] National Council On Interpreting in Helath Care: https://www.ncihc.org/. Accessed: 2020-05-07.

[58] National Organisation on Disability 2005. Report on special needs assessment for Katrina evacuees (SNAKE) project. http://www.katrinadisability.info/PDFsK/ katrina_snake_report.pdf.

[59] Norton, J., Raturi, A., Nardi, B., Prost, S., McDonald, S., Pargman, D., Bates, O., Normark, M., Tomlinson, B., Herbig, N. and Dombrowski, L. 2017. A grand challenge for HCI: Food + sustainability. Interactions. (2017). DOI:https://doi.org/ $10.1145 / 3137095$

[60] O'Brien, J. 2017. First-of-its-kind Trial Goes Plaintiff's Way; Winn-Dixie Must Update Website For The Blind. Forbes.

[61] On The Pandemic: What The Coronavirus Outbreak Reveals About Ableism At Georgetown: 2020. https://georgetownvoice.com/2020/04/22/on-the-pandemicwhat-the-coronavirus-outbreak-reveals-about-ableism-at-georgetown

[62] Oswal, S.K. 2019. Disability, ICT and elearning platforms: Faculty-facing embedded work tools in learning management systems. ASSETS 2019 - 21st International ACM SIGACCESS Conference on Computers and Accessibility. (2019), 105-111. DOI:https://doi.org/10.1145/3308561.3355620.

[63] Palen, L. and Liu, S.B. 2007. Citizen Communications in Crisis: Anticipating a Future of ICT-Supported Public Participation. Proceedings of the SIGCHI Conference on Human Factors in Computing Systems (New York, NY, USA, 2007), 727-736.

[64] Prieto, N. 2014. Home delivery services improve accessibility of food, medications, and medical care for people with transportation issues. EasterSeals Crossroads: taking on disability together.

[65] Profita, H.P., Stangl, A., Matuszewska, L., Sky, S. and Kane, S.K. 2016. Nothing to hide: Aesthetic customization of hearing AIDS and cochlear implants in an online community. ASSETS 2016 - Proceedings of the 18th International ACM SIGACCESS Conference on Computers and Accessibility. (2016), 219-227. DOI:https: //doi.org/10.1145/2982142.2982159.

[66] Roberts, J.B., Crittenden, L.A. and Crittenden, J.C. 2011. Students with disabilities and online learning: A cross-institutional study of perceived satisfaction with accessibility compliance and services. Internet and Higher Education. 14, 4 (2011), 242-250. DOI:https://doi.org/10.1016/j.iheduc.2011.05.004.

[67] Ryan, F. and Marsh, S. 2020. Disabled people cut off from vital supplies due to panic buying. The Guardian.

[68] Sanchez-Gordon, S. and Luján-Mora, S. How Could MOOCs Become Accessible? The Case of edX and the Future of Inclusive Online Learning.

[69] Scholz, F., Yalcin, B. and Priestley, M. 2017. Internet access for disabled people: Understanding socio-relational factors in Europe. Cyberpsychology: fournal of Psychosocial Research on Cyberpspace. 11, 1 (2017)

[70] Schraefel, M.C. 2013. Green food through green food: A human centered design approach to green food technology. UbiComp 2013 Adjunct - Adjunct Publication of the 2013 ACM Conference on Ubiquitous Computing (2013), 595-598.

[71] Seale, J. and Cooper, M. 2010. E-learning and accessibility: An exploration of the potential role of generic pedagogical tools. Computers and Education. 54, 4 (2010), 1107-1116. DOI:https://doi.org/10.1016/j.compedu.2009.10.017.

[72] Secretary DeVos Reiterates Learning Must Continue for All Students, Declines to Seek Congressional Waivers to FAPE, LRE Requirements of IDEA: 2020. 
https://www.newsbreak.com/news/1555578539187/secretary-devos-reiterateslearning-must-continue-for-all-students-declines-to-seek-congressional-waiversto-fape-lre-requirements-of-idea.

[73] Shinohara, K., Kawas, S., Ko, A.J. and Ladner, R.E. 2018. Who teaches accessibility? A survey of U.S. computing faculty. SIGCSE 2018 - Proceedings of the 49th ACM Technical Symposium on Computer Science Education (Feb. 2018), 197-202.

[74] Social-Emotional Learning should be priority during COVID-19 crisis: 2020 http://neatoday.org/2020/04/15/social-emotional-learning-during-covid/.

[75] Sohaib, O. and Kang, K. 2017. E-commerce web accessibility for people with disabilities. Complexity in Information Systems Development. Springer. 87-100.

[76] Speech Reading: https://www.cdc.gov/ncbddd/hearingloss/parentsguide/building/ speech-reading.html. Accessed: 2020-05-07.

[77] Storer, K.M. and Branham, S.M. 2019. "That's the way sighted people do it": What blind parents can teach technology designers about co-reading with children.
DIS 2019 - Proceedings of the 2019 ACM Designing Interactive Systems Conference. (2019), 385-398. DOI:https://doi.org/10.1145/3322276.3322374.

[78] Takayama, K. 2017. Disaster Relief and Crisis Intervention with Deaf Communities: Lessons Learned from the Japanese Deaf Community. Fournal of Social Work in Disability and Rehabilitation. 16, 3-4 (2017), 247-260. DOI:https: //doi.org/10.1080/1536710X.2017.1372241.

[79] Vieweg, S. and Starbird, K. 2010. Microblogging in Mass Emergency. Distribution (2010), 1-4.

[80] When Coronavirus Care Gets Lost in Translation: 2020. https://www.nytimes.com/ 2020/04/17/health/covid-coronavirus-medical-translators.html. Accessed: 202005-07.

[81] White, B. 2014. Disaster Relief for Deaf Persons: Lessons from Hurricanes Katrina and Rita. Review of Disability Studies: An International fournal. 2, 3 (2014). 